\title{
Síndrome de embolia gordurosa: relato de caso associado à lipoaspiração*
}

\author{
Jõao Carlos Folador ${ }^{1}$, Gabriela Elisete Bier², Renata Ferreira de Camargo², Mônicca Sperandio²
}

Mulher, 40 anos, 72 horas após lipossucção apresentou dispnéia súbita, febre, taquipnéia e taquicardia. Radiografia torácica e eco-doppler de membros inferiores eram normais, mas hipoperfusão pulmonar à cintilografia sugeriu tromboembolismo pulmonar. A pós três dias de heparinização, observou-se hipóxia, anemia e confusão mental. Diagnosticou-se síndrome de embolia gordurosa, suspendeu-se heparinização e iniciou-se pulsoterapia com corticóide, seguida de melhora clínica e alta hospitalar.

(J Pneumol 1999;25(2):114-117)

\section{Fat embolism syndrome: report of a case associated to liposuction}

A forty year old woman, 72 hours after liposuction, complained of sudden dyspnea, fever and tachycardia. Thoracic radiography and duplex-scan of deep veins were normal. Pulmonary scintigraphy suggested thromboembolism. The patient had hypoxia, anemia and mental confusion after three days of systemic heparin. Once fat embolism syndrome was suspected, heparin infusion was stopped and corticosteroid therapy was started. After that, the patient was much better and discharged from hospital.

Descritores - Síndrome de embolia gordurosa. Tromboembolismo pulmonar. Lipoaspiração.

Key words - Fat embolism syndrome. Pulmonary thromboembolism. Liposuction.

\section{INTRODUÇÃO}

A primeira descrição de síndrome de embolia gordurosa (SEG) foi realizada após achado em autópsia por Zenter ${ }^{(1)}$, em 1861. Após 12 anos, Von Bergman realizou seu primeiro diagnóstico clínico(2). Embora esta síndrome seja reconhecida por mais de 100 anos, sua fisiopatologia permanece incompleta e ainda persistem discussões sobre seu diagnóstico específico e manejo clínico.

* Trabalho realizado no Serviço de Pneumologia - Clínica Médica do Hospital de Caridade Santa Casa de Misericórdia de Curitiba.

1. Chefe do Serviço de Pneumologia. Professor responsável pela Disciplina de Pneumologia na Pontifícia Universidade Católica do Paraná.

2. Médica residente do Serviço de Clínica Médica.

Endereço para correspondência - J oão Carlos Folador, H ospital Santa Casa de Misericórdia de Curitiba, Serviço de Clínica M édica, 20 andar, Praça Rui Barbosa, 694 - 80010-030 - Curitiba, PR. Tel. (041) 3203543.

Recebido para publicação em 4/9/98. Reapresentado em 4/ 12/98. A provado, após revisão, em 2/2/99.
Siglas e abreviaturas utilizadas neste trabalho

SEG - Síndrome de embolia gordurosa

SEG é caracterizada pelo aparecimento de ácidos graxos ou gordura livre na circulação sanguínea, pulmões, cérebro, rins e outros órgãos. A tríade clássica caracterizada por insuficiência respiratória aguda com infiltrado pulmonar difuso, disfunção neurológica e rash com petéquias ocorre em apenas $0,5 \%$ a $2,0 \%$ dos $\operatorname{casos}^{(3)}$.

Em $90 \%$ dos casos, a SEG ocorre após trauma brusco complicado por fraturas de ossos longos, geralmente de membros inferiores. Raramente ocorre em associação com osteomielite, pancreatite aguda, diabetes melito, anemia falciforme, queimaduras, reconstruções articulares, lipoaspiração, bypass cardiopulmonar, e infusão parenteral de lipídios(4,5).

O objetivo desse relato é apresentar a associação de SEG à lipoaspiração, uma causa incomum da doença e dificuldades do seu diagnóstico.

\section{RELATO DO CASO}

Paciente do sexo feminino, branca, 40 anos, 72 horas após a realização de procedimento cirúrgico (histerectomia, ooforectomia e lipoaspiração) apresentou-se com dispnéia súbita e febre, evoluindo com piora progressiva em 48 horas para dispnéia aos pequenos esforços, hipertermia e mal-estar. Estava em tratamento hormonal para endometriose há 


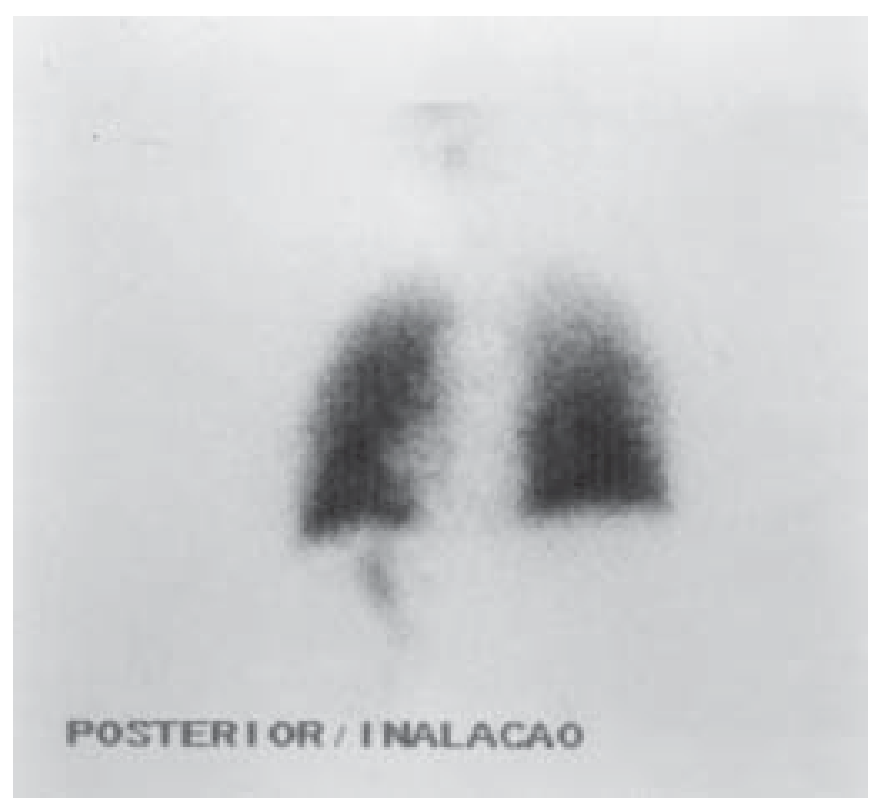

Figura 1 - Cintilografia pulmonar de inalação

seis meses, negava uso de outras medicações. Sem história de tabagismo e etilismo.

A o exame observou-se estado geral comprometido, taquipnéia (freqüência respiratória de 30ipm), taquicardia, palidez cutâneo-mucosa $(++/$ IV $)$ e hipertermia $\left(39^{\circ} \mathrm{C}\right)$. Presença de estertores finos em $1 / 3$ médio posterior de hemitórax esquerdo à ausculta pulmonar. Restante do exame físico sem particularidades, incluíndo ausência de petéquias e fundoscopia normal.

A gasometria arterial demonstrou $\mathrm{PO}_{2}$ de $59,9 \mathrm{mmH} \mathrm{g} \mathrm{em}$ ar ambiente. 0 hemograma apresentava anemia normocrômica normocítica (hemoglobina de $8,2 \mathrm{~g} / \mathrm{dl}$ e volume globular de $25,1 \mathrm{ml} / \mathrm{dl}$ ), leucócitos de 8.400 (16,9\% de linfócitos e ausência de bastonetes), plaquetas de 142.000 e velocidade de hemossedimentação de $113 \mathrm{~mm}$.

A primeira hipótese diagnóstica foi de tromboembolismo pulmonar. Realizou-se radiografia de tórax, que demonstrou pulmões com transparência normal, vascularização pulmonar normal, área cardíaca dentro dos padrões normais de acordo com a razão cardiotorácica; a seguir, o eco-doppler de membros inferiores não evidenciou trombose venosa profunda, com veias safenas pérvias, havendo limitação da técnica à realização da avaliação pélvica.

Cintilografia pulmonar observou ao estudo de inalação (figura 1) alargamento da fissura interlobar entre os lobos inferior e superior do lobo esquerdo. Estudo de perfusão (figura 2 ), com diminuição do radiofármaco no segmento superior do lobo inferior do pulmão esquerdo, sugeriu alta probabilidade de tromboembolismo pulmonar.

Iniciou-se heparinização endovenosa e, após três dias de tratamento, o quadro clínico não apresentou melhora, com

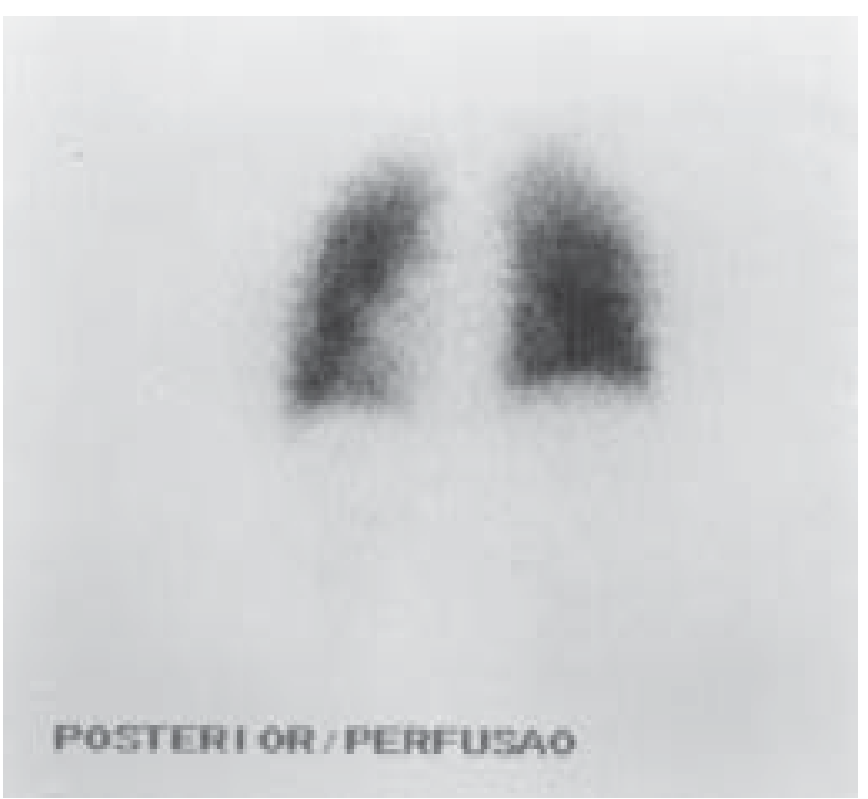

Figura 2 - Cintilografia pulmonar de perfusão

persistência da febre, piora progressiva da dispnéia, hipoxemia, anemia e confusão mental que não apresentava relação direta com os episódios de hipertermia.

U ma vez excluída hipótese de infecção, iniciou-se pulsoterapia com corticóide (metilprednisolona 7,5 mg/ kg EV a cada 6 horas, totalizando 12 doses). Houve melhora clínica evidente, seguida de alta hospitalar com diagnóstico de síndrome de embolia gordurosa.

\section{DısCUSSÃo}

Lipossucção é um procedimento seguro; entretanto, não deve ser indicado em todas as circunstâncias. Indica-se nos casos em que há gordura localizada, pacientes jovens e com boas condições de pele. Não é indicada para obesidade generalizada e pacientes com doenças associadas devem ser cuidadosamente avaliados(6).

Taxa de complicações em lipossucção é de cerca de 5 a $10 \%$ dos casos, constituindo-se principalmente por anestesia, seroma, edema, pigmentação, dor e hematoma ${ }^{(7)}$. Complicações pulmonares após lipossucção incluem redução da capacidade pulmonar total, capacidade vital e capacidade residual funcional, hipoxemia leve e atelectasia parcial. Tromboembolismo pulmonar é raro, mas pode ocorrer após esse procedimento $^{(8)}$.

SEG ocorre mais freqüente após fratura de ossos longos. A composição da gordura no tecido adiposo e nos ossos parece ser semelhante. $\mathrm{Na}$ lipossucção ocorre trauma extensivo, com ruptura de vasos sanguíneos e compressão do tecido adiposo.

O mecanismo da embolia gordurosa parece ser devido a gordura neutra proveniente da área lesada, que emboliza para 
circulação pulmonar, onde é degradada pelos pneumócitos em ácidos graxos livres, que são quimicamente mais lesivos ao parênquima pulmonar, levando a insuficiência respiratória(6).

É importante distinguir embolia gordurosa de síndrome de embolismo gorduroso. Embolia gordurosa refere-se a presença de glóbulos de gordura no parênquima pulmonar e circulação periférica após fratura de um osso longo ou trauma maior. Síndrome de embolia gordurosa é uma manifestação mais séria do embolismo gorduroso, que envolve insuficiência respiratória progressiva, trombocitopenia e deterioração do estado mental, ocorrendo usualmente 72 horas após a lesão ${ }^{(9)}$. Embolismo gorduroso é comum e pode ser detectado em mais de $90 \%$ dos pacientes com fratura de ossos longos. Síndrome de embolia gordurosa é menos comum, ocorrendo em $0,5 \%$ a $2,0 \%$ dos pacientes com fratura de ossos longos isolada e $5 \%$ a $10 \%$ em pacientes com múltiplas lesões em ossos longos ou concomitantes fraturas pélvi$\operatorname{cas}^{(9,10)}$.

O padrão clínico da SEG envolve uma distribuição bimodal. É incomum o curso fulminante, que leva a falência de múltiplos órgãos, no qual os primeiros sintomas surgem cerca de 12 horas após a lesão, apresentando maior mortalidade pelo envolvimento neurológico proeminente, que leva ao coma e embolização maciça, com falência de ventrículo direito e colapso cardíaco. A maioria dos pacientes apresenta um curso mais progressivo, com sintomas em 24 a 72 horas após lesão, cuja gravidade é variável, desde casos leves com hipóxia transitória e confusão, até síndrome de insuficiência respiratória e coma(9).

Diagnóstico da SEG é um diagnóstico de exclusão, baseado no quadro clínico(3). Os sintomas iniciais são freqüentemente inespecíficos, como hipertermia leve e taquicardia. A insuficiência respiratória leva a hipoxemia em $30 \%$ dos pacientes. Sintomas neurológicos ocorrem em $60-70 \%$ dos casos e tendem a seguir as alterações pulmonares, manifestando-se através de desorientação, confusão, estupor ou coma ${ }^{(11)}$. Petéquias aparecem em $40-50 \%$ dos pacientes, são freqüentes no pescoço, axila, tronco e conjuntiva e podem ser evanescentes ${ }^{(4)}$. A biópsia de pele nas lesões petequiais, pode revelar gordura intravascular em $90 \%$ dos casos. Fundoscopia revela microinfartos em retina ${ }^{(11)}$.

O presente caso manifestou-se com clínica compatível com $S E G$, iniciando com quadro inespecífico (dispnéia, hipertermia e mal-estar), evoluíndo com piora respiratória, hipóxia, anemia e confusão mental; não houve aparecimento de petéquias e a fundoscopia foi normal.

Os critérios clássicos de Gurd(12) ainda são usados para ajudar no diagnóstico da SEG (tabela 1), sendo necessário pelo menos um critério maior e três menores ou dois critérios maiores e dois menores. Richards(11), em seu estudo, sugere ser difícil diferenciar SEG de outros tipos de doenças pulmonares baseando-se apenas nesses critérios.
No presente caso, a paciente apresentava um critério maior (hipóxia) e três critérios menores (taquicardia, febre e anemia inexplicada).

Exames laboratoriais para identificar embolismo gorduroso têm especificidade e sensibilidade baixas. Hipóxia arterial é comum, anemia e trombocitopenia podem estar presentes. Produtos da degradação do fibrinogênio, tempo de protrombina, velocidade de hemossedimentação e nível de C5 estão freqüentemente aumentados $s^{(9,11)}$.

Testes biológicos como atividade de lipase sérica ou pesquisa de glóbulos de gordura na urina, sangue, escarro ou líquor apresentam especificidade pobre. Lipidúria ocorre em muitos traumas em que os pacientes não manifestam SEG. Pesquisa de lipídios dentro de macrófagos alveolares no lavado broncoalveolar pode ser um procedimento acurado e rápido para o diagnóstico dessa síndrome ${ }^{(13,14)}$.

Inclusões de lipídios em células alveolares são freqüentes durante insuficiência respiratória de qualquer etiologia (traumática ou não traumática). Entretanto, determinação da percentagem de células do lavado broncoalveolar e contagem de vacúolos de gordura pode contribuir para o diagnóstico de SEG em pacientes com trauma mecânico-ventilatório, com falência respiratória ${ }^{(13)}$.

Castella ${ }^{(15)}$ demostrou que um cateter de Swan-Ganz pode ajudar no diagnóstico, pela citologia microvascular pulmonar, técnica nova que analisa os capilares pulmonares obtidos pelo cateter.

Radiografias seriadas podem mostrar infiltrados pulmonares, apesar dessas imagens não serem específicas. Cintilografia de ventilação-perfusão ajuda na exclusão de tromboembolismo pulmonar ${ }^{(9)}$. No caso em questão a cintilografia pulmonar demonstrou hipoperfusão no segmento superior do lobo inferior do pulmão esquerdo, indicando alta probabilidade de tromboembolismo pulmonar, o que corroborou no erro de diagnóstico inicial.

TABELA 1

Critérios de $\mathrm{G}$ urd ${ }^{(12)}$ para diagnóstico

de síndrome de embolia gordurosa

\section{Critérios}

\section{Maiores}

Hipóxia $\left(\mathrm{PaO}_{2}<60 \mathrm{mmHg}\right.$ e $\left.\mathrm{FiO}_{2}>0,4\right)$

Depressão do sistema nervoso central Petéquias

\section{Menores}

Taquicardia (freqüência cardíaca > 120bpm)

Febre (temperatura $>39^{\circ} \mathrm{C}$ )

Trombocitopenia (contagem de plaquetas $<150 \times 10^{9} / \mathrm{L}$ )

Glóbulos de gordura na urina ou escarro

Embolia para retina

Anemia inexplicada 
0 manejo desses pacientes é baseado em suporte primário, incluindo suporte ventilatório e monitorização em unidade intensiva se necessário. Tratamentos históricos, como heparina, álcool intravenoso, dextran de baixo peso molecular, albumina e glicose hipertônica com insulina, falharam em mostrar benefícios ${ }^{(9)}$. Terapia com corticosteróides foi primeiro sugerida por Ashbaugh e Petty(16), em 1966; foram seguidos estudos em animais demonstrando que o corticóide atenua a lesão pulmonar induzida por ácidos graxos livres. Alho e colaboradores ${ }^{(17)}$ mostraram benefício com corticóide como terapêutica profilática para pacientes com alto risco de SEG. Porém ainda permanece controversa essa indicação de corticoterapia ${ }^{(9)}$.

0 mecanismo de ação dos corticóides envolve proteção do endotélio capilar, estabilização de membranas dos granulócitos, redução da ativação do sistema complemento, diminuição da agregação plaquetária e liberação de serotoninas ${ }^{(11)}$. Muitos médicos recomendam a administração de corticóides pela sua ação antiinflamatória, aceitando as complicações que podem ocorrer pelo uso dos mesmos.

SEG é uma doença importante pela sua significante morbidade e potencial mortalidade. Nas décadas de 1960 e 1970 a mortalidade por SEG era de $10 \%$ a $20 \%$; em anos mais recentes, ocorreu redução para $5 \%$ a $10 \%{ }^{(18)}$.

\section{Conclusão}

Lipossucção é uma técnica efetiva e segura para escultura do corpo, mas não é isenta de riscos. SEG é uma de suas complicações pulmonares e sua incidência pode ser maior do que a esperada previamente. 0 seu diagnóstico é difícil pelo quadro clínico inespecífico e pela falta de exames com alta especificidade e sensibilidade. A diferenciação de SEG com tromboembolismo pulmonar é importante, podendo ocorrer dúvida no diagnóstico entre essas duas entidades, mas o manejo terapêutico é diferente para cada doença e o atraso no início do tratamento correto aumenta a morbimortalidade em ambos os casos.

\section{REFERÊNCIAS}

1. Zenker FA. Beitrage zur A natomie und Physiologie de Lung. Dresden, Germany: J Braunsdorf, 1861.

2. Von Bergman EB. Ein Fall todlicher fettemboli. Berl Klin Wochenschr 1873;10:385.

3. J ohson MJ , Lucas GL. Fat embolism syndrome. Orthopedics 1996; 19:41-48.

4. Fabian TC. Unraveling the fat embolism syndrome. N Engl J Med 1993;32:961-963.

5. Fabian TC, Hoots AV, Stanford DS, Patterson CR, Mangiante EC. Fat embolism syndrome: prospective evaluation in 92 fracture patients. Crit Care Med 1990;18:42-46.

6. Ross RM, J ohnson GW. Fat embolism after liposuction. Chest 1988; 93:1294-1295.

7. Pitman H, Teimorian B. Suction lipectomy: complications and results by survey. Plast Reconstr Surg 1985;76:65-69.

8. Hunter GR, Crapo R, Broadbent T, Woolf R. Pulmonary complications following abdominal lipectomy. Plast Reconstr Surg 1983;71: 809-817.

9. Bulger EM, Smith DG, Maier RV, J urkovich GJ. Fat embolim syndrome: a 10 year review. Arch Surg 1997;132:435-439.

10. Muller $C$, Rahn B, Pfiser U, Meinig R. The incidence, pathogenesis, diagnosis, and treatment of fat embolism. Orthop Rev 1994;23:107 117.

11. Richards RR. Fat embolism syndrome. JCC 1997;40:334-339.

12. Gurd A. Fat embolism: an aid to diagnosis. J Bone J oint Surg $[\mathrm{Br}]$ 1970;52:732-737.

13. Mimoz O, Edouard A, Quillard J, Verra F, Fleury J. Contribution of bronchoalveolar lavage to the diagnosis of posttraumatic pulmonary fat embolism. Intensive Care Med 1995;21:973-980.

14. Vedrinne J M, Guillaume C, Gagnieu MC, Gratadour P, Fleuret C, Motin $\mathrm{J}$. Bronchoalveolar lavage in trauma patients for diagnosis of fat embolism syndrome. Chest 1992;102:1323-1327.

15. Castella X, Valles J, Cabezuelo MA, Fernandez R, Artigas A. Fat embolism syndrome and pulmonary microvascular cytology. Chest 1992; 101:1710-1711.

16. A shbaugh D, Petty T. The use of corticosteroids in the treatment of respiratory failure associated with massive fat embolism. Surg Ginecol Obstet 1966;123:493-500

17. Alho A, Saikku K, Eerola P, Koskinen M, Hamalainen M. Corticosteroids in patients with a high risk of fat embolism syndrome. Surg Gynecol Obstet 1978;147:358-362.

18. Fulde G, Harrison P. Fat embolism: a review. Arch Emerg Med 1991; 8:233-239. 